\title{
SYNTHESIS, STRUCTURAL CHARACTERIZATION OF SOME PYRAZOLO [1-5A] PYRIMIDINE AND IMIDAZO[1,2-B]- PYRAZOLE DERIVATIVES AS ANTI-CANCER ACTIVITY
}

\author{
Usama Fathy $^{1, \bowtie}$, Ameen A. Abu-Hashem ${ }^{2}$, Rasha S. Gouhar ${ }^{3}$, Hanem M. \\ Awad $^{4}$ and Abdelbaset M. Elgamal ${ }^{5}$ \\ ${ }^{1}$ Applied Organic Chemistry Department, National Research Centre, 33 El Bohouth st. (former \\ EL Tahrir st.)-Dokki-Giza-Egypt-P.O.12622. \\ ${ }^{2}$ Photochemistry Department (Heterocyclic Unit), National Research Centre, 33 El Bohouth st. \\ (former EL Tahrir st.)-Dokki-Giza-Egypt-P.O.12622. \\ ${ }^{3}$ Therapeutic Chemistry Department, National Research Centre, 33 El Bohouth st. (former EL \\ Tahrir st.)-Dokki-Giza-Egypt-P.O.12622. \\ ${ }^{4}$ Tanning Materials and Leather Technology Department, National Research Centre, 33 El \\ Bohouth st. (former EL Tahrir st.)-Dokki-Giza-Egypt-P.O.12622. \\ ${ }^{5}$ Chemistry of Natural and Microbial Products Department, National Research Centre, 33 El \\ Bohouth st. (former EL Tahrir st.)-Dokki-Giza-Egypt-P.O.12622. \\ ${ }^{\square}$ Corresponding Author: usamafathy2000@yahoo.com
}

\begin{abstract}
For anticancer cell line evaluation, a new sequence of pyrazolo [1-5a] pyrimidine and imidazo[1,2-b] pyrazole derivatives were synthesized. The reaction of 5-aminopyrazolo with appropriate functionalized enaminones derivatives and 2-bromoacetophenone to afford, respectively. In vitro tests were performed on the newly synthesized compounds against HepG-2 and MCF-7 cancer cells to assay their cytotoxicity. A few compounds showed promising activity as anticancer agents against MCF-7 cancer cell.
\end{abstract}

Keywords: Pyrazole, Pyrimidine, Imidazole, Enaminone, Anticancer Activity.

RASĀYAN J. Chem., Vol. 14, No.2, 2021

\section{INTRODUCTION}

Cancer is a term used to describe a group of cancerous diseases that can affect various parts of the body. ${ }^{1}$ The liver can develop primary cancer, in which it forms itself, or to cancer that develops elsewhere in the body and then is transferred to the liver (secondary or metastatic cancer). In addition, breast cancer is cancer that forms in the cells of the breasts. As a result, there is a constant need for new chemotherapeutic medicines for cancer care.Pyrazolo[1,5-a] pyrimidine is one of the pyrazoleopyrimidine isomers, they are purine analogue ${ }^{2}$. They have a wide variety of biological activity such as anti-proliferative agents ${ }^{3}$, antifungal $^{4}$, antimicrobial ${ }^{5}, 6$, chronic myeloid leukemia inhibitors ${ }^{7}$, antioxidant ${ }^{8,9}$, cathepsin $\mathrm{K}$ inhibitors ${ }^{10,11}$, anti-tubercular ${ }^{12}$, anti-inflammatory ${ }^{13}$, antivira $1^{14}$. Currently, they reported strong cytotoxicity against several human cancer cell lines. ${ }^{15-20}$

For example, compound I showed the highest activity against HepG2, MCF7, HCT116 and HeLa cell lines with $\mathrm{IC}_{50}$ values $1.88,0.47,0.54$ and $0.40 \mu \mathrm{g} / \mathrm{ml}$, respectively ${ }^{21}$. Also, Compound II was found to be the most active, with $\mathrm{IC}_{50}$ values of $1.98,2.20$, and $2.61 \mathrm{M}$, respectively, against MCF-7, BT474, and A549 cancer cell lines. Compounds III and IV were also shown to have anticancer activity against four separate cell lines (HepG2, MCF-7, A549 and Caco2). ${ }^{22}$

Imidazo[1,2-b] pyrazole derivatives were synthesized previously by many authors. ${ }^{23-25}$ In the last years, it was reported as an anticancer against. ${ }^{26,27}$ For example, compounds V, VI and VII show promise as an anticancer agent with an $\mathrm{IC}_{50}$ less than $10 \mathrm{mM}$ in the 6 cell lines tested. ${ }^{27}$ 

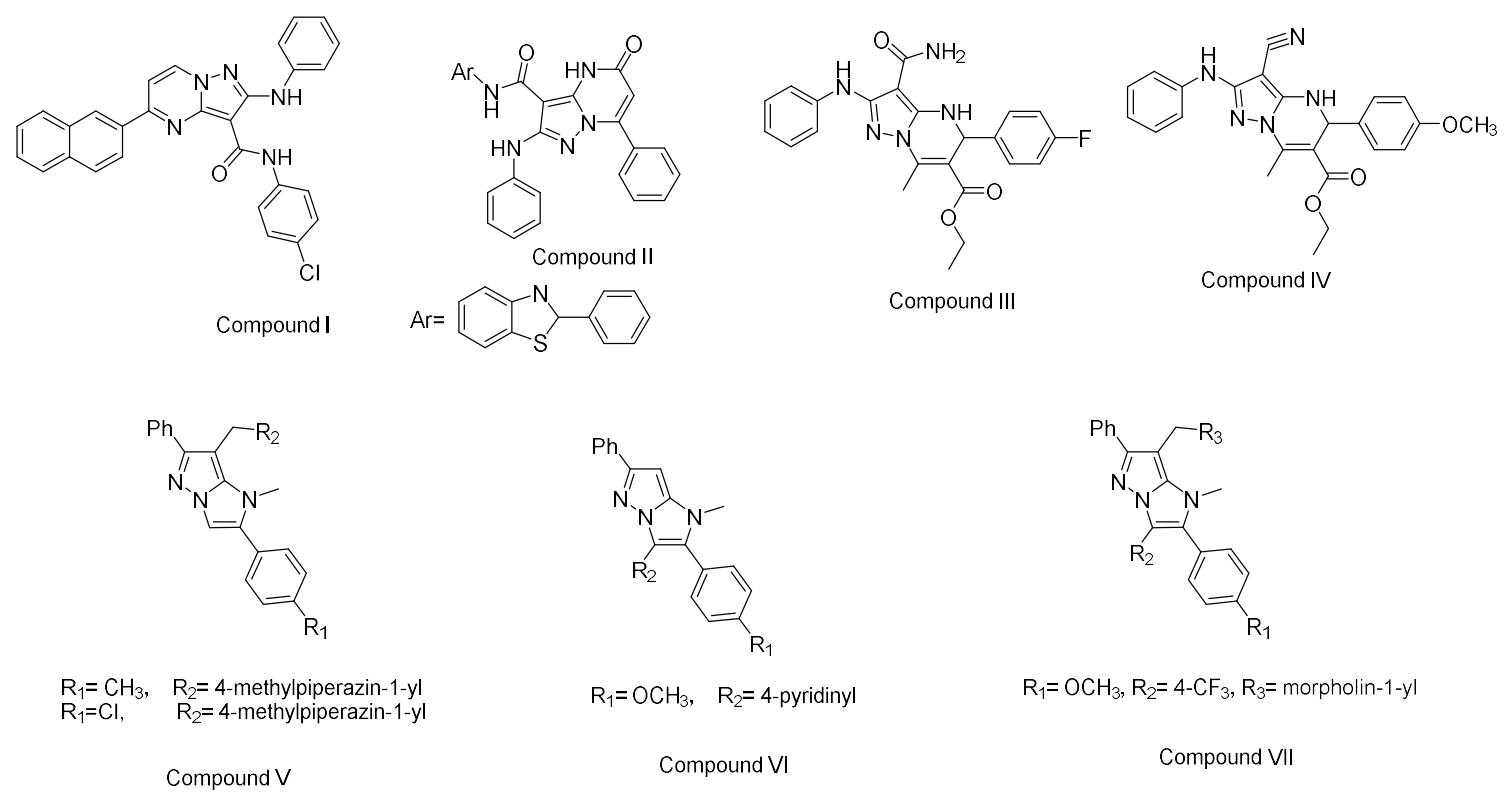

Fig.-1: Structure of Some Anticancer Pyrazolo[1-5a]pyrimidin and Imidazo[1,2-b]pyrazole Derivatives

By screening HepG-2 and MCF-7 for anticancer activity, compounds 10a-l and 12a-c derivatives carrying a phenyldiazenyl moiety have been prepared to produce new potent anticancer agents.

\section{EXPERIMENTAL}

\section{Synthesis of 1-phenyl-2-bromoethanone (2-bromoacetophenone) (2)}

Add anhydrous $\mathrm{AlCl}_{3}(0.5 \mathrm{~g})$ to a stirred solution of acetophenone $(12.0 \mathrm{~g}, 0.1 \mathrm{~mol})$ in dry ether $(100 \mathrm{ml})$. Bromine ( $16.0 \mathrm{~g}, 0.2 \mathrm{~mol}$ ) was added drop-wise over $30 \mathrm{~min}$. duration with stirring for $20 \mathrm{~min}$. to the resulting solution, the reaction mixture was poured on cold water. Separated the organic layer, washed twice with water, then with a solution of $\mathrm{NaHCO}_{3}(5 \%, 150 \mathrm{ml})$ and gradually dried over anhydrous $\mathrm{Na}_{2} \mathrm{SO}_{4}$, then filtered. Under reduced pressure, the solvent was extracted and the solid residue was recrystallized from ether to provide compound 2.

\section{Synthesis of 3-oxo-3-phenylpropanenitrile (3)}

$\mathrm{KCN}$ solution $\left(6.5 \mathrm{~g}, 0.1 \mathrm{~mol}\right.$ in $\left.30 \mathrm{ml} \mathrm{H}_{2} \mathrm{O}\right)$ was added to a solution of phenacyl bromide $(10 \mathrm{~g}, 0.05 \mathrm{~mol})$ in benzene $(60 \mathrm{ml})$ and the reaction mixture was heated to $70{ }^{\circ} \mathrm{C}$ with intensive shaking until the aqueous layer become deep orange. The reaction mixture was cooled and the aqueous layer was removed, benzene washed and $\mathrm{HCl}$ acidified. Filtration, collected the solid precipitate, washed with cold water, dried and finally recrystallized from hot $\mathrm{H}_{2} \mathrm{O}$ to produce compound 3 .

\section{Synthesis of 2-oxo-N,2-diphenylacetohydrazonoyl cyanide (6)}

In EtOH $(100 \mathrm{ml}) \mathrm{CH}_{3} \mathrm{COONa} .3 \mathrm{H}_{2} \mathrm{O}(13.6 \mathrm{~g}, 0.1 \mathrm{~mol})$ was introduced to a stirred solution of benzoylacetonitrile (3) $(14.5 \mathrm{~g}, 0.1 \mathrm{~mol})$. The mixture was chilled at $0^{\circ} \mathrm{C}$ after stirring for $15 \mathrm{~min}$ and treated with a cold solution of the required arylamine $(0.1 \mathrm{~mol})$ in $6 \mathrm{M} \mathrm{HCl}(6 \mathrm{ml})$ with $\mathrm{NaNO}_{2}(0.7 \mathrm{~g}, 10 \mathrm{mmol})$ in $5 \mathrm{ml} \mathrm{H} \mathrm{H}_{2} \mathrm{O}$. The diazonium salt was added and stirred at $0-5^{\circ} \mathrm{C}$ for an additional $2 \mathrm{~h}$, then placed in a refrigerator $\left(4^{\circ} \mathrm{C}\right)$ for $8 \mathrm{~h}$. The resulting solid was stored, washed thoroughly with $\mathrm{H}_{2} \mathrm{O}$ and dried by filtration. To give the corresponding acetohydrazonoyl cyanide (6), the crude product was crystallized from EtOH.

\section{Synthesis of 3-phenyl-4-(phenyldiazenyl)-1H-pyrazol-5-amine derivatives (7a-c)}

Compounds (7a-c) were obtained through the reaction of acetohydrazonoyl cyanide derivatives (6a-c) with $98 \% \mathrm{NH}_{2}-\mathrm{NH}_{2} \cdot \mathrm{H}_{2} \mathrm{O}$ in $\mathrm{n}-\mathrm{BuOH}$ and $4 \mathrm{~h}$ reflux. To afford compounds (7a-c), the product obtained was filtered, dried and crystallized by EtOH. 
RASĀYAN J. Chem.

Vol. 14 | No. 2 |741-750| April - June | 2021

3-phenyl-4-(phenyldiazenyl)-1H-pyrazol-5-amine (7a)

Yield (71\%), m.p. $217^{\circ} \mathrm{C}$; IR $\left(\mathrm{KBr}, v \mathrm{~cm}^{1}\right): 3330-3195\left(\mathrm{NH}+\mathrm{NH}_{2}\right),{ }^{1} \mathrm{H}$ NMR (DSMO- $\left.d_{6}, \mathrm{ppm}\right) \delta 6.2(\mathrm{~s}$, $2 \mathrm{H}, \mathrm{D}_{2} \mathrm{O}$-exchangeable, $\left.\mathrm{NH}_{2}\right), 7.30-7.51(\mathrm{~m}, 6 \mathrm{H}, 3 \mathrm{H} \mathrm{Ar}+3 \mathrm{H} \mathrm{ph}), 7.73(\mathrm{~d}, J=7.8 \mathrm{~Hz}, 2 \mathrm{H}(o), \mathrm{Ar}), 8.13(\mathrm{~d}$, $J=8.5 \mathrm{~Hz}, 2 \mathrm{H}(o), \mathrm{ph}), 12.09$ (s, 1H, $\mathrm{D}_{2} \mathrm{O}$-exchangeable, NH); ${ }^{13} \mathrm{C}$ NMR (DSMO-d6, ppm) 95.35, 126.30, $127.39,127.93,128.33,128.34,128.89(2 \mathrm{C}), 129.14(2 \mathrm{C}), 132.02,139.86,145.22,149.84,153.51$; MS $\left(\mathrm{C}_{15} \mathrm{H}_{13} \mathrm{~N}_{5}\right),(\mathrm{m} / \mathrm{z}, 100 \%)=263\left(\mathrm{M}^{+}\right)$.

\section{4-((4-chlorophenyl)diazenyl)-3-phenyl-1H-pyrazol-5-amine (7b)}

Yield (78\%); m.p. $230{ }^{\circ} \mathrm{C}$; IR $\left(\mathrm{KBr}, v \mathrm{~cm}^{1}\right)$ : 3335-3197( $\left.\mathrm{NH}+\mathrm{NH}_{2}\right) ;{ }^{1} \mathrm{H}$ NMR (DSMO- $\left.d_{6}, \mathrm{ppm}\right) \delta 6.31(\mathrm{~s}$, $2 \mathrm{H}, \mathrm{D}_{2} \mathrm{O}$-exchangeable, $\left.\mathrm{NH}_{2}\right), 7.38-7.53(\mathrm{~m}, 4 \mathrm{H}, \mathrm{Ar}), 7.74(\mathrm{t}, J=8.7 \mathrm{~Hz}, 3 \mathrm{H}(p, m), \mathrm{ph}), 8.10(\mathrm{~d}, J=8.5$ $\mathrm{Hz}, 2 \mathrm{H}(o), \mathrm{ph}), 12.11$ (s, 1H, $\mathrm{D}_{2} \mathrm{O}$-exchangeable, NH); ${ }^{13} \mathrm{C}$ NMR (DSMO-d6, ppm) 96.50, 126.27, 127.42, $127.99,128.34,128.47(2 \mathrm{C}), 128.83,128.93,129.15,131.81,132.90,141.18,145.47,151.88$; MS $\left(\mathrm{C}_{15} \mathrm{H}_{12} \mathrm{ClN}_{5}\right),(m / z, \%)=297\left(\mathrm{M}^{+}, 35\right), 399\left(\mathrm{M}^{+}+2,11\right)$.

\section{4-((4-flurophenyl)diazenyl)-3-phenyl-1H-pyrazol-5-amine (7c)}

Yield (70\%), m.p. $225^{\circ} \mathrm{C}$; IR $\left(\mathrm{KBr}, v \mathrm{~cm}^{1}\right): 3335-3200\left(\mathrm{NH}+\mathrm{NH}_{2}\right),{ }^{1} \mathrm{H}$ NMR (DSMO- $\left.d_{6}, \mathrm{ppm}\right) \delta 6.2(\mathrm{~s}, 2 \mathrm{H}$, $\mathrm{D}_{2} \mathrm{O}$-exchangeable, $\left.\mathrm{NH}_{2}\right), 7.28-7.47(\mathrm{~m}, 4 \mathrm{H}(m, o), \mathrm{Ar}-), 7.77(\mathrm{~m}, 3 \mathrm{H}(p, m) \mathrm{ph}), 8.11(\mathrm{~d}, J=8.5 \mathrm{~Hz}, 2 \mathrm{H}(o)$, ph), 12.08 (s, 1H, $\mathrm{D}_{2} \mathrm{O}$-exchangeable, NH); ${ }^{13} \mathrm{C}$ NMR (DSMO-d6, ppm) 95.60, 115.73, 116.04, 122.52, $127.37,127.87,128.26,128.86,130.55,131.88,133.02,140.99,147.42,149.84,158.40 ; \mathrm{MS}\left(\mathrm{C}_{15} \mathrm{H}_{12} \mathrm{FN}_{5}\right)$, $(\mathrm{m} / \mathrm{z}, 100 \%)=281\left(\mathrm{M}^{+}\right)$.

Synthesis of 2,7-diphenyl-3-(phenyldiazenyl) pyrazolo[1,5-a] pyrimidine derivatives (10a-l)

A mixture of compound $7(\approx 0.3 \mathrm{~g} ; 0.001 \mathrm{~mol})$ and the corresponding enaminones $(\mathbf{8}),(0.001 \mathrm{~mol})$ in 20 $\mathrm{ml} \mathrm{CH}_{3} \mathrm{COOH}$ was refluxed for $1-2 \mathrm{~h}$, left to cool. The product obtained was washed, dried, and crystallized from $\mathrm{EtOH}$ to give 10a-l compounds, respectively.

\section{2,7-diphenyl-3-(phenyldiazenyl)pyrazolo[1,5-a]pyrimidine (10a)}

Yield (72\%), m.p. $190^{\circ} \mathrm{C}$; IR $\left(\mathrm{KBr}, v \mathrm{~cm}^{1}\right)$ : $1660(\mathrm{C}=\mathrm{N}),{ }^{1} \mathrm{H}$ NMR (DSMO- $\left.d_{6}, \mathrm{ppm}\right) \delta 7.45-7.65(\mathrm{~m}, 10 \mathrm{H}$,$) ,$ $7.80(\mathrm{~d}, J=7.5 \mathrm{~Hz}, 2 \mathrm{H}), 8.15(\mathrm{~d}, J=6.5 \mathrm{~Hz}, 2 \mathrm{H}), 8.20(\mathrm{~d}, J=1.5 \mathrm{~Hz}, 2 \mathrm{H}), 8.86(\mathrm{~d}, J=4.5 \mathrm{~Hz}, 1 \mathrm{H}$ of pyrimidine); ${ }^{13} \mathrm{C}$ NMR (DSMO-d6, ppm) 110.93, 120.85, 121.81, 124.81, 128.63(2C), 128.69(2C), 129.45(2C), 129.50(2C), 129.59(2C), 129.99(2C), 131.50, 131.80, 139.62, 144.68, 146.13, 153.55, 153.78, $153.83 ; \mathrm{MS}\left(\mathrm{C}_{24} \mathrm{H}_{17} \mathrm{~N}_{5}\right),(\mathrm{m} / \mathrm{z}, 100 \%)=375\left(\mathrm{M}^{+}\right)$.

\section{7-(4-chlorophenyl)-2-phenyl-3-(phenyldiazenyl)pyrazolo[1,5-a]pyrimidine (10b)}

Yield (72\%), m.p. $203^{\circ} \mathrm{C}$; IR $\left(\mathrm{KBr}, v \mathrm{~cm}^{1}\right): 1665(\mathrm{C}=\mathrm{N}),{ }^{1} \mathrm{H}$ NMR (DSMO- $\left.d_{6}, \mathrm{ppm}\right) \delta 7.48-7.59(\mathrm{~m}, 7 \mathrm{H}$,$) ,$ $7.70(\mathrm{~d}, J=8.1 \mathrm{~Hz}, 2 \mathrm{H}), 7.79(\mathrm{~d}, J=8.1 \mathrm{~Hz}, 2 \mathrm{H}), 8.16(\mathrm{~d}, J=7.5 \mathrm{~Hz}, 2 \mathrm{H}), 8.22(\mathrm{~d}, J=8.4 \mathrm{~Hz}, 2 \mathrm{H}), 8.87$ (d, $J=4.2 \mathrm{~Hz}, 1 \mathrm{H}$ of pyrimidine); ${ }^{13} \mathrm{C}$ NMR (DSMO-d6, ppm) 110.73, 120.56, 120.60, 121.63, 124.68, 128.46, 128.63, 128.63, 129.27, 129.32(2C), 129.46(2C), 129.90(2C), $131.55(2 \mathrm{C}), 131.67,136.15,139.44$, 144.76, 153.28, 153.58, 153.62; $\mathrm{MS}\left(\mathrm{C}_{24} \mathrm{H}_{16} \mathrm{ClN}_{5}\right),(\mathrm{m} / z, 100 \%)=409\left(\mathrm{M}^{+}, 28\right), 411\left(\mathrm{M}^{+}+2,9\right)$.

\section{2-phenyl-3-(phenyldiazenyl)-7-(p-tolyl)pyrazolo[1,5-a]pyrimidine (10c)}

Yield $(72 \%)$, m.p. $180^{\circ} \mathrm{C}$; IR $\left(\mathrm{KBr}, v \mathrm{~cm}^{1}\right): 1664(\mathrm{C}=\mathrm{N}),{ }^{1} \mathrm{H}$ NMR (DSMO- $\left.d_{6}, \mathrm{ppm}\right) \delta 2.45\left(\mathrm{~s}, 3 \mathrm{H}, \mathrm{CH}_{3}\right)$ 7.46-7.60 (m, 9H,), $7.80(\mathrm{~d}, J=7 \mathrm{~Hz}, 2 \mathrm{H}), 8.13(\mathrm{~d}, J=8.1 \mathrm{~Hz}, 2 \mathrm{H}), 8.17(\mathrm{~d}, J=1.2 \mathrm{~Hz}, 2 \mathrm{H}), 8.87(\mathrm{~d}, J=$ $4.2 \mathrm{~Hz}, 1 \mathrm{H}$ of pyrimidine); ${ }^{13} \mathrm{C}$ NMR (DSMO-d6, ppm) 20.97, 110.30, 120.62, 121.53(2C), 126.93, 128.38(2C), 129.05(2C), 129.20(2C), 129.24(2C), 129.34(2C), 129.68(2C), 129.75 (2C), 131.64, 141.50, $146.00,153.51,153.60 ; \mathrm{MS}\left(\mathrm{C}_{25} \mathrm{H}_{19} \mathrm{~N}_{5}\right),(\mathrm{m} / z, 100 \%)=389\left(\mathrm{M}^{+}\right)$.

\section{2-Phenyl-3-(phenyldiazenyl)-7-(thiophen-2-yl)pyrazolo[1,5-a]pyrimidine (10d)}

Yield $(72 \%)$, m.p. $140^{\circ} \mathrm{C}$; IR $\left(\mathrm{KBr}, v \mathrm{~cm}^{1}\right): 1668(\mathrm{C}=\mathrm{N}),{ }^{1} \mathrm{H}$ NMR (DSMO- $\left.d_{6}, \mathrm{ppm}\right) \delta 7.41-7.58(\mathrm{~m}, 7 \mathrm{H}$,$) ,$ $7.81(\mathrm{~d}, J=1.5 \mathrm{~Hz}, 2 \mathrm{H}), 7.98(\mathrm{~d}, J=4.8 \mathrm{~Hz}, 1 \mathrm{H}), 8.20(\mathrm{~d}, J=4.8 \mathrm{~Hz}, 1 \mathrm{H}), 8.37(\mathrm{~d}, J=7.5 \mathrm{~Hz}, 2 \mathrm{H}), 8.60$ $(\mathrm{d}, J=3.6 \mathrm{~Hz}, 1 \mathrm{H}$ of pyrimidine $), 8.82(\mathrm{~d}, J=4.8 \mathrm{~Hz}, 1 \mathrm{H}$ of pyrimidine $) ;{ }^{13} \mathrm{C}$ NMR (DSMO-d6, ppm) 21.17, 
RASĀYAN J. Chem.

Vol. 14 | No. 2 |741-750| April - June | 2021

$106.82,121.82$, 124.73, 127.97, 128.85(2C), 129.45(2C), 129.48(2C), 129.85(2C), 130.05, 131.71, 133.17, 136.24, 139.44, 139.53, 152.90, 153.18, 153.80, 172.14; $\mathrm{MS}\left(\mathrm{C}_{22} \mathrm{H}_{15} \mathrm{~N}_{5} \mathrm{~S}\right),(\mathrm{m} / \mathrm{z}, 100 \%)=381\left(\mathrm{M}^{+}\right)$.

\section{3-((4-chlorophenyl)diazenyl)-2,7-diphenylpyrazolo[1,5-a]pyrimidine (10e)}

Yield $(72 \%)$, m.p. $170^{\circ} \mathrm{C}$; IR $\left(\mathrm{KBr}, v \mathrm{~cm}^{1}\right)$ : $1665(\mathrm{C}=\mathrm{N}),{ }^{1} \mathrm{H}$ NMR (DSMO- $\left.d_{6}, \mathrm{ppm}\right) \delta 7.53-7.68(\mathrm{~m}, 8 \mathrm{H}$, ), $7.79(\mathrm{~d}, J=7.5 \mathrm{~Hz}, 2 \mathrm{H}), 7.79(\mathrm{~d}, J=8.1 \mathrm{~Hz}, 2 \mathrm{H}), 8.14(\mathrm{~d}, J=7.5 \mathrm{~Hz}, 2 \mathrm{H}), 8.17(\mathrm{~d}, J=1.8 \mathrm{~Hz}, 1 \mathrm{H}$ of pyrimidine), 8.90 (d, $J=1.2 \mathrm{~Hz}, 1 \mathrm{H}$ of pyrimidine); ${ }^{13} \mathrm{C}$ NMR (DSMO-d6, ppm) 111.02, 114.95, 123.25, 124.64, 128.30, 128.54, 128.57(2C), 129.41(2C), 129.54(2C), 129.67(2C), 129.89(2C), 131.42 (2C), $131.52,134.18,146.15,152.23,153.67,153.98 ; \mathrm{MS}\left(\mathrm{C}_{24} \mathrm{H}_{16} \mathrm{ClN}_{5}\right),(\mathrm{m} / \mathrm{z}, 100 \%)=409\left(\mathrm{M}^{+}, 23\right), 411\left(\mathrm{M}^{+}+2\right.$, $7)$.

7-(4-chlorophenyl)-3-((4-chlorophenyl)diazenyl)-2-phenylpyrazolo[1,5-a]pyrimidine (10f)

Yield $(72 \%)$, m.p. $198^{\circ} \mathrm{C}$; IR $\left(\mathrm{KBr}, v \mathrm{~cm}^{1}\right): 1670(\mathrm{C}=\mathrm{N}),{ }^{1} \mathrm{H}$ NMR (DSMO- $\left.d_{6}, \mathrm{ppm}\right) \delta 7.50(\mathrm{~d}, J=8.1 \mathrm{~Hz}$, $\left.2 \mathrm{H}, o-\mathrm{Ar}_{1}\right), 7.60\left(\mathrm{~d}, J=4.5 \mathrm{~Hz}, 2 \mathrm{H}, m-\mathrm{Ar}_{1}\right), 7.72-7.75\left(\mathrm{~m}, 5 \mathrm{H}, 2 \mathrm{H} \mathrm{Ar} \mathrm{A}_{2}+3 \mathrm{H} \mathrm{ph}\right), 7.76(\mathrm{~d}, J=8.1 \mathrm{~Hz}, 2 \mathrm{H}, o-$ ph), $8.12\left(\mathrm{~d}, J=7.5 \mathrm{~Hz}, 2 \mathrm{H} o-\mathrm{Ar}_{2}\right), 8.21(\mathrm{~d}, J=8.1 \mathrm{~Hz}, 1 \mathrm{H}$ of pyrimidine $), 8.86(\mathrm{~d}, J=0.9 \mathrm{~Hz}, 1 \mathrm{H}$ pyrimidine); ${ }^{13} \mathrm{C}$ NMR (DSMO-d6, ppm) 110.92, 123.23, 124.65, 128.29, 128.49, 128.58, 128.63(2C), 129.36(2C), 129.54(2C), 131.43(2C), 131.54(2C), 131.74(2C), 134.21, 136.21, 144.84, 152.16, 153.55, 153.84; $\mathrm{MS}\left(\mathrm{C}_{24} \mathrm{H}_{15} \mathrm{Cl}_{2} \mathrm{~N}_{5}\right),(\mathrm{m} / z, 100 \%)=443\left(\mathrm{M}^{+}, 21\right), 445\left(\mathrm{M}^{+}+2,12\right), 447\left(\mathrm{M}^{+}+4,2.5\right)$.

\section{3-((4-chlorophenyl)diazenyl)-2-phenyl-7-(p-tolyl)pyrazolo[1,5-a]pyrimidine (10g)}

Yield (72\%), m.p. $180^{\circ} \mathrm{C}$; IR (KBr, $\left.v \mathrm{~cm}^{1}\right): 1665(\mathrm{C}=\mathrm{N}),{ }^{1} \mathrm{H}$ NMR (DSMO- $\left.d_{6}, \mathrm{ppm}\right) \delta 2.45\left(\mathrm{~s}, 3 \mathrm{H}, \mathrm{CH}_{3^{-}}\right)$, 7.47-7.64 (m, 9H), $7.80(\mathrm{~d}, J=8.7 \mathrm{~Hz}, 2 \mathrm{H}), 8.13(\mathrm{~d}, J=8.1 \mathrm{~Hz}, 2 \mathrm{H}), 8.18(\mathrm{~d}, J=2.1 \mathrm{~Hz}, 1 \mathrm{H}$ of pyrimidine $)$, 8.89 (d, $J=4.5 \mathrm{~Hz}, 1 \mathrm{H}$ of pyrimidine) ${ }^{13} \mathrm{C}$ NMR (DSMO-d6, ppm) 21.14, 110.73, 120.66, 120.73, 121.26, $123.29,126.96,128.34,128.60(2 \mathrm{C}), 128.72,128.88,129.21(2 \mathrm{C}), 129.42,129.48,129.61,129.89,131.58$, 134.20, 141.72, 145.43, 145.54, 146.27, 152.27, 153.98; $\mathrm{MS}\left(\mathrm{C}_{25} \mathrm{H}_{18} \mathrm{ClN}_{5}\right),(\mathrm{m} / z, 100 \%)=423\left(\mathrm{M}^{+}, 26\right)$, $425\left(\mathrm{M}^{+}+2,8\right)$.

\section{3-((4-chlorophenyl)diazenyl)-2-phenyl-7-(thiophen-2-yl)pyrazolo[1,5-a]pyrimidine (10h)}

Yield (72\%), m.p. $230^{\circ} \mathrm{C}$; IR $\left(\mathrm{KBr}, v \mathrm{~cm}^{1}\right)$ : $1668(\mathrm{C}=\mathrm{N}),{ }^{1} \mathrm{H}$ NMR (DSMO- $\left.d_{6}, \mathrm{ppm}\right) \delta 7.42-7.65(\mathrm{~m}, 6 \mathrm{H})$, $7.82\left(\mathrm{~d}, J=8.1 \mathrm{~Hz}, 2 \mathrm{H}\right.$ of $\left.\mathrm{Ar}_{1}\right), 8.04(\mathrm{~d}, J=4.8 \mathrm{~Hz}, 1 \mathrm{H}$ of thiophen $), 8.23$ (d, $J=5.1 \mathrm{~Hz}, 1 \mathrm{H}$ of thiophen-), $8.36(\mathrm{~d}, J=7.8 \mathrm{~Hz}, 2 \mathrm{H}$ of ph), $8.63(\mathrm{~d}, J=3.9 \mathrm{~Hz}, 1 \mathrm{H}$ of pyrimidine $), 8.85(\mathrm{~d}, J=5.1 \mathrm{~Hz}, 1 \mathrm{H}$ of pyrimidine $)$; ${ }^{13} \mathrm{C}$ NMR (DSMO-d6, ppm) 94.30, 106.98, 120.69, 123.34, 127.99, 128.85(2C), 129.29(2C), 129.41(2C), $129.53(2 \mathrm{C}), 129.90,130.64,131.49,133.28,134.27,136.38,144.48,152.31,153.14 ; \mathrm{MS}\left(\mathrm{C}_{22} \mathrm{H}_{14} \mathrm{ClN}_{5} \mathrm{~S}\right)$, $(\mathrm{m} / z, 100 \%)=415\left(\mathrm{M}^{+}, 16\right), 425\left(\mathrm{M}^{+}+2,5\right)$.

\section{3-((4-fluorophenyl)diazenyl)-2,7-diphenylpyrazolo[1,5-a]pyrimidine (10i)}

Yield (72\%), m.p. $120^{\circ} \mathrm{C}$; IR $\left(\mathrm{KBr}, v \mathrm{~cm}^{1}\right)$ : $1670(\mathrm{C}=\mathrm{N}),{ }^{1} \mathrm{H}$ NMR (DSMO- $\left.d_{6}, \mathrm{ppm}\right) \delta 7.37-7.68(\mathrm{~m}, 8 \mathrm{H})$, $7.84(\mathrm{~d}, J=5.4 \mathrm{~Hz}, 2 \mathrm{H}), 7.87(\mathrm{~d}, J=5.4 \mathrm{~Hz}, 2 \mathrm{H}), 8.14(\mathrm{~d}, J=7.5 \mathrm{~Hz}, 2 \mathrm{H}), 8.19$ (d, $J=2.5 \mathrm{~Hz}, 1 \mathrm{H}$ of pyrimidine), 8.88 (d, $J=4.5 \mathrm{~Hz}, 1 \mathrm{H}$ of pyrimidine); ${ }^{13} \mathrm{C}$ NMR (DSMO-d6, ppm) 94.24, 110.87, 115.64, $116.07,116.38,121.78,123.65,124.47,128.20,128.53,128.57,128.82,129.35,129.49,129.65,129.86$, $131.40,131.57,139.45,146.06,150.35,150.67,161.22,164.50(\mathrm{C}-\mathrm{F}) ; \mathrm{MS}\left(\mathrm{C}_{24} \mathrm{H}_{16} \mathrm{FN}_{5}\right),(\mathrm{m} / \mathrm{z}, 100 \%)=393$ $\left(\mathrm{M}^{+}\right)$.

\section{7-(4-Chlorophenyl)-3-((4-fluorophenyl)diazenyl)-2-phenylpyrazolo[1,5-a]pyrimidine (10j)}

Yield (72\%), m.p. $130^{\circ} \mathrm{C}$; IR $\left(\mathrm{KBr}, v \mathrm{~cm}^{1}\right): 1673(\mathrm{C}=\mathrm{N}),{ }^{1} \mathrm{H}$ NMR (DSMO- $\left.d_{6}, \mathrm{ppm}\right) \delta 7.34(\mathrm{dd}, J=2.1 \mathrm{~Hz}$, $2 \mathrm{H}), 7.37(\mathrm{dd}, J=8.4 \mathrm{~Hz}, 2 \mathrm{H}), 7.69(\mathrm{~d}, J=1.5 \mathrm{~Hz}, 2 \mathrm{H}), 8.14(\mathrm{~d}, J=7.5 \mathrm{~Hz}, 2 \mathrm{H}), 7.81(\mathrm{~d}, J=1.8 \mathrm{~Hz}, 2 \mathrm{H})$, $8.12(\mathrm{~d}, J=5.4 \mathrm{~Hz}, 2 \mathrm{H}), 8.21(\mathrm{~d}, J=6.9 \mathrm{~Hz}, 2 \mathrm{H}), 8.85\left(\mathrm{~d}, J=4.8 \mathrm{~Hz}, 1 \mathrm{H}\right.$ of pyrimidine); ${ }^{13} \mathrm{C}$ NMR (DSMOd6, ppm) 95.12, 110.80, 116.05, 116.35, 123.68, 123.89, 124.53, 128.51, 128.65, 128.82, 129.37, 129.51, $129.65,129.86,131.53,131.75,136.21,139.43,144.79,150.34,153.37,153.66,161.26,164.50$ (C-F); MS $\left(\mathrm{C}_{24} \mathrm{H}_{15} \mathrm{ClFN}_{5}\right),(\mathrm{m} / z, 100 \%)=427\left(\mathrm{M}^{+}, 22\right), 429\left(\mathrm{M}^{+}+2,7\right)$. 


\section{RASĀYAN $J$. Chem. \\ Vol. 14 | No. 2 |741-750| April - June | 2021}

\section{3-((4-Fluorophenyl)diazenyl)-2-phenyl-7-(p-tolyl)pyrazolo[1,5-a]pyrimidine (10k)}

Yield (72\%), m.p. $183^{\circ} \mathrm{C}$; IR $\left(\mathrm{KBr}, v \mathrm{~cm}^{1}\right)$ : $1665(\mathrm{C}=\mathrm{N}),{ }^{1} \mathrm{H}$ NMR (DSMO- $\left.d_{6}, \mathrm{ppm}\right) 2.44\left(\mathrm{~s}, 3 \mathrm{H}, \mathrm{CH}_{3}-\right)$, 7.35-7.65 (m, 9H), $7.82(\mathrm{~d}, J=8.5 \mathrm{~Hz}, 2 \mathrm{H}), 8.15(\mathrm{~d}, J=7.5 \mathrm{~Hz}, 2 \mathrm{H}), 8.17(\mathrm{~d}, J=2.1 \mathrm{~Hz}, 1 \mathrm{H}$ of pyrimidine $)$, $8.85\left(\mathrm{~d}, J=4.5 \mathrm{~Hz}, 1 \mathrm{H}\right.$ of pyrimidine); ${ }^{13} \mathrm{C}$ NMR (DSMO-d6, ppm) 21.03, 110.62, 120.13, 121.50, 123.45, 126.96, 128.51, 128.71(2C), 128.80, 129.32(2C), 129.45, 129.63, 129.88, 129.92, 131.72, 135.20, 141.13, 144.86, 145.66, 146.53, 153.21, 163.98; $\mathrm{MS}\left(\mathrm{C}_{24} \mathrm{H}_{18} \mathrm{FN}_{5}\right),(\mathrm{m} / \mathrm{z}, 100 \%)=407\left(\mathrm{M}^{+}\right)$.

\section{3-((4-Fluorophenyl)diazenyl)-2-phenyl-7-(thiophen-2-yl)pyrazolo[1,5-a]pyrimidine (101)}

Yield $(72 \%)$, m.p. $200^{\circ} \mathrm{C}$; IR $\left(\mathrm{KBr}, v \mathrm{~cm}^{1}\right): 1670(\mathrm{C}=\mathrm{N}),{ }^{1} \mathrm{H}$ NMR (DSMO- $\left.d_{6}, \mathrm{ppm}\right) \delta 7.38-7.62(\mathrm{~m}, 6 \mathrm{H})$, $7.82(\mathrm{~d}, J=5.4 \mathrm{~Hz}, 2 \mathrm{H}), 7.95(\mathrm{~d}, J=4.8 \mathrm{~Hz}, 1 \mathrm{H}), 8.18(\mathrm{~d}, J=5.1 \mathrm{~Hz}, 1 \mathrm{H}), 8.32(\mathrm{~d}, J=7.2 \mathrm{~Hz}, 2 \mathrm{H}), 8.56$ (d, $J=3.9 \mathrm{~Hz}, 1 \mathrm{H}$ of pyrimidine), $8.78\left(\mathrm{~d}, J=4.8 \mathrm{~Hz}, 1 \mathrm{H}\right.$ of pyrimidine); ${ }^{13} \mathrm{C}$ NMR (DSMO-d6, ppm) $106.66,116.04,116.34,123.66,123.78,124.42,127.80(2 \mathrm{C}), 128.67(2 \mathrm{C}), 129.32(2 \mathrm{C}), 129.68(2 \mathrm{C})$, 130.52(2C), 131.54(2C), 133.00, 136.05, 139.37, 144.13, 150.37, 152.70, 153.03, 161.23, 164.52, 171.99; $\mathrm{MS}\left(\mathrm{C}_{22} \mathrm{H}_{14} \mathrm{FN}_{5} \mathrm{~S}\right),(\mathrm{m} / z, 100 \%)=309\left(\mathrm{M}^{+}\right)$.

\section{Synthesis of $1 \mathrm{H}$-imidazo[1,2-b]pyrazole Derivatives (12a-c)}

For a mixture of compound 7a-c $(0.26 \mathrm{~g}, 1 \mathrm{mmol}), \mathrm{K}_{2} \mathrm{CO}_{3}(0.45,3 \mathrm{mM})$ and compound $\mathbf{2}(0.357 \mathrm{~g}, 1 \mathrm{mmol})$ in acetone $(25 \mathrm{ml})$, the reaction mixture was refluxed for $4 \mathrm{~h}$. and cooled at room temperature. The product was filtered, washed, dried, and recrystallized from EtOH to give 13a-b compounds, respectively.

\section{2,6-Diphenyl-7-(phenyldiazenyl)-1H-imidazo[1,2-b]pyrazole (12a)}

Yield (72\%), m.p. $250^{\circ} \mathrm{C}$; IR (KBr, $\left.v \mathrm{~cm}^{1}\right): 3360(\mathrm{NH}),{ }^{1} \mathrm{H}$ NMR (DSMO- $\left.d_{6}, \mathrm{ppm}\right) \delta 7.15-7.65(\mathrm{~m}, 10 \mathrm{H})$, $7.77(\mathrm{~d}, J=5.4 \mathrm{~Hz}, 2 \mathrm{H}), 7.95(\mathrm{~d}, J=4.8 \mathrm{~Hz}, 2 \mathrm{H}), 8.45\left(\mathrm{~s}, 1 \mathrm{H}\right.$ of imidazo pyrazole), $12.32\left(\mathrm{~s}, 1 \mathrm{H}, \mathrm{D}_{2} \mathrm{O}-\right.$ exchangeable, NH), ${ }^{13} \mathrm{C}$ NMR (DSMO-d6, ppm) 98.13, 119.85, 125.41, 125.97, 127.12(2C), 128.22, 128.36(2C), 128.78(2C), 128.97(2C), 129.53(2C), 129.98(2C), 130.52(2C), 133.11, 139.50, 141.27, 149.15; $\mathrm{MS}\left(\mathrm{C}_{23} \mathrm{H}_{17} \mathrm{~N}_{5}\right),(\mathrm{m} / z, 100 \%)=363\left(\mathrm{M}^{+}\right)$.

\section{7-((4-Chlorophenyl)diazenyl)-2,6-diphenyl-1H-imidazo[1,2-b]pyrazole (12b)}

Yield (72\%), m.p. $270^{\circ} \mathrm{C}$; IR $\left(\mathrm{KBr}, v \mathrm{~cm}^{1}\right): 3370(\mathrm{NH}),{ }^{1} \mathrm{H}$ NMR (DSMO- $\left.d_{6}, \mathrm{ppm}\right) \delta 7.35-7.67(\mathrm{~m}, 10 \mathrm{H})$, $7.80(\mathrm{~d}, J=5.4 \mathrm{~Hz}, 2 \mathrm{H}), 7.96(\mathrm{~d}, J=4.8 \mathrm{~Hz}, 2 \mathrm{H}), 8.48\left(\mathrm{~s}, 1 \mathrm{H}\right.$ of imidazo pyrazole), $12.52\left(\mathrm{~s}, 1 \mathrm{H}, \mathrm{D}_{2} \mathrm{O}-\right.$ exchangeable, NH), ${ }^{13} \mathrm{C}$ NMR (DSMO-d6, ppm) 98.15, 120.30, 127.21, 128.14(2C), 128.44, 128.50, 128.78(2C), 128.95(2C), 128.97(2C), 129.65(2C), 130.88(2C), 131.51, 132.62, 134.71, 140.72, 141.80, 150.10; $\mathrm{MS}\left(\mathrm{C}_{23} \mathrm{H}_{16} \mathrm{ClN}_{5}\right),(\mathrm{m} / z, 100 \%)=397\left(\mathrm{M}^{+}, 26\right), 399\left(\mathrm{M}^{+}+2,8\right)$.

\section{7-((4-Fluorophenyl)diazenyl)-2,6-diphenyl-1H-imidazo[1,2-b]pyrazole(12c)}

Yield $(72 \%)$, m.p. $>300^{\circ} \mathrm{C}$; IR $\left(\mathrm{KBr}, v \mathrm{~cm}^{1}\right): 3375(\mathrm{NH}),{ }^{1} \mathrm{H}$ NMR (DSMO- $\left.d_{6}, \mathrm{ppm}\right) \delta 7.45-7.68(\mathrm{~m}, 10 \mathrm{H})$, $7.82(\mathrm{~d}, J=5.4 \mathrm{~Hz}, 2 \mathrm{H}), 7.95(\mathrm{~d}, J=4.8 \mathrm{~Hz}, 2 \mathrm{H}), 8.50(\mathrm{~s}, 1 \mathrm{H}$ of imidazo pyrazole $), 12.78\left(\mathrm{~s}, 1 \mathrm{H}, \mathrm{D}_{2} \mathrm{O}-\right.$ exchangeable, $\mathrm{NH}$ ), ${ }^{13} \mathrm{C}$ NMR (DSMO-d6, ppm) 98.22, 116.50, 120.31, 123.7, 127.92 (2C), 128.15, 128.33, 128.47, 128.85(2C), 128.97, 129.65(2C), 130.88(2C), 131.71(2C), 134.50, 140.88, 141.80, 150.10, 161.12; $\mathrm{MS}\left(\mathrm{C}_{23} \mathrm{H}_{16} \mathrm{ClN}_{5}\right),(\mathrm{m} / 2,100 \%)=381\left(\mathrm{M}^{+}\right)$.

\section{General Procedure}

The melting points were uncorrected and determined using an Electrothermal IA 9100 (Shimadzu, Japan) apparatus. Vario El-Mentar apparatus (Shimadzu, Japan), National Research Centre, Cairo, Egypt, carried out microanalytical research. IR spectra were reported on a Perkin-Elmer 1650 Spectrophotometer, National Research Centre, Cairo, Egypt as potassium bromide pellets using $\mathrm{KBr}$ disc technique. NMR experiments in Deuterated Dimethylsulphoxide (DMSO-d6) were calculated on a Varian $-300 \mathrm{MHz}$ and chemical changes were expressed as parts per million; ppm ( $\pi$ values) against TMS as an internal standard, Faculty of Science, University of Cairo, Cairo, Egypt. Mass spectra were recorded on the $70 \mathrm{eV}$ mass spectrometer at Shimadzu GCMS-QP-1000EX, University of Cairo, Cairo, Egypt. 
RASĀYAN J. Chem.

Vol. 14 | No. 2 |741-750| April - June | 2021

\section{Biological Evaluation}

\section{Cell Culture Conditions}

The American Model Culture Collection (Rockville, MD) obtained the cells of human liver carcinoma (HepG-2), and human breast adenocarcinoma (MCF-7). The cytotoxicity activities on the lines of human cancer cells HepG-2 and MCF-7 were calculated using MTT assay, which was based on the reduction of tetrazolium salt in viable cells by mitochondrial dehydrogenases ${ }^{28-30}$ The absorbance was measured at 570 $\mathrm{nm}$, using a microplate reader from SpectraMax ${ }^{\circledR}$ Paradigm ${ }^{\circledR}$ Multi-Mode. The relative viability of the cells was expressed as the mean percentage of viable cells compared to the control cells not treated.

\section{Statistical Analysis}

Both tests were carried out in triplicate and replicated on three separate days. All the values were as mean \pm SD. SPSS probit testing software system (SPSS Inc., Chicago, IL) calculated the $\mathrm{IC}_{50}$.

\section{Chemistry}

\section{RESULTS AND DISCUSSION}

In the first scheme, the phenacyl bromide was obtained by bromination of acetophenone, then reacted with $\mathrm{KCN}$ in dry benzene to afford compound $\mathbf{3}$, which allowed to reacting with 1-chloro-2-phenyldiazene (5) to yield compound $\mathbf{6}$, when it reacting with $\mathrm{NH}_{2} \cdot \mathrm{NH}_{2} \cdot \mathrm{H}_{2} \mathrm{O}$ yield 5-aminopyrazole derivatives (7a-c) The IR spectrum of compound 7a-c displayed the characteristic sharp absorption bands of $\mathrm{NH}$ and $\mathrm{NH}_{2}$ at $\approx 3335$, $3200 \mathrm{~cm}-1$, respectively (Scheme-1). The ${ }^{1} \mathrm{H}-\mathrm{NMR}$ spectrum of 7a-c exhibited the singlet signals of protons $\mathrm{NH}_{2}$ and $\mathrm{NH}$ appear at 6.2-6.31 and 12.08-12.11 ppm, respectively. 5-aminopyrazole derivatives (7a-c) as an intermediate key for synthesis the synthesis of compounds 10a-l and 12a-c derivatives as shown in Scheme-2, and 3.

$$
\begin{aligned}
& \underset{\text { ether }}{\stackrel{\mathrm{Br}_{2} / \mathrm{AlCl}_{3}}{\longrightarrow}} \\
& \begin{array}{l|ll|}
\hline 7 & \mathrm{Ar} \\
\hline \mathrm{a} & \mathrm{Ph} & \\
\mathrm{b} & 4-\mathrm{ClC}_{6} \mathrm{H}_{4} & \\
\mathrm{c} & 4-\mathrm{FC}_{6} \mathrm{H}_{4}
\end{array}
\end{aligned}
$$

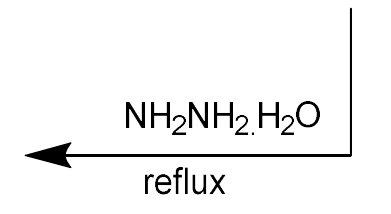

Scheme-1: The Mechanistic Pathway for the Synthesis of 5-amino phenyldiazenyl-1H-pyrazol Derivatives (7a-c)

In Scheme-2, mating occurs between 5-amino phenyldiazenyl-1 $H$-pyrazol (7a-c) and functionalized enaminones (8) by refluxing in glacial $\mathrm{CH}_{3} \mathrm{COOH}$, the addition of nucleophilic amino group to the olefinic double bond and releasing dimethylamine led to the formation of the corresponding pyrazolo [1-5a] pyrimidine (10a-l) (Scheme-2). The ${ }^{1} \mathrm{H}-\mathrm{NMR}$ spectrum of 10a-l exhibited the disappearance of the singlet as duplet signals at $\delta \approx 8.17-8.56$ and $\delta \approx 8.85-8.90 \mathrm{ppm}$, respectively. The ${ }^{13} \mathrm{C}-\mathrm{NMR}$ spectrum of 10a-I 
RASĀYAN J. Chem.

Vol. 14 | No. 2 |741-750| April - June | 2021

signals of protons $\mathrm{NH}_{2}$ and $\mathrm{NH}$ and revealed deshielding of $\mathrm{H}-5, \mathrm{H}-6$ of pyrazolo [1-5a] pyrimidine appear exhibited a high chemical shift of F-C where it appears at about 164-171 ppm.

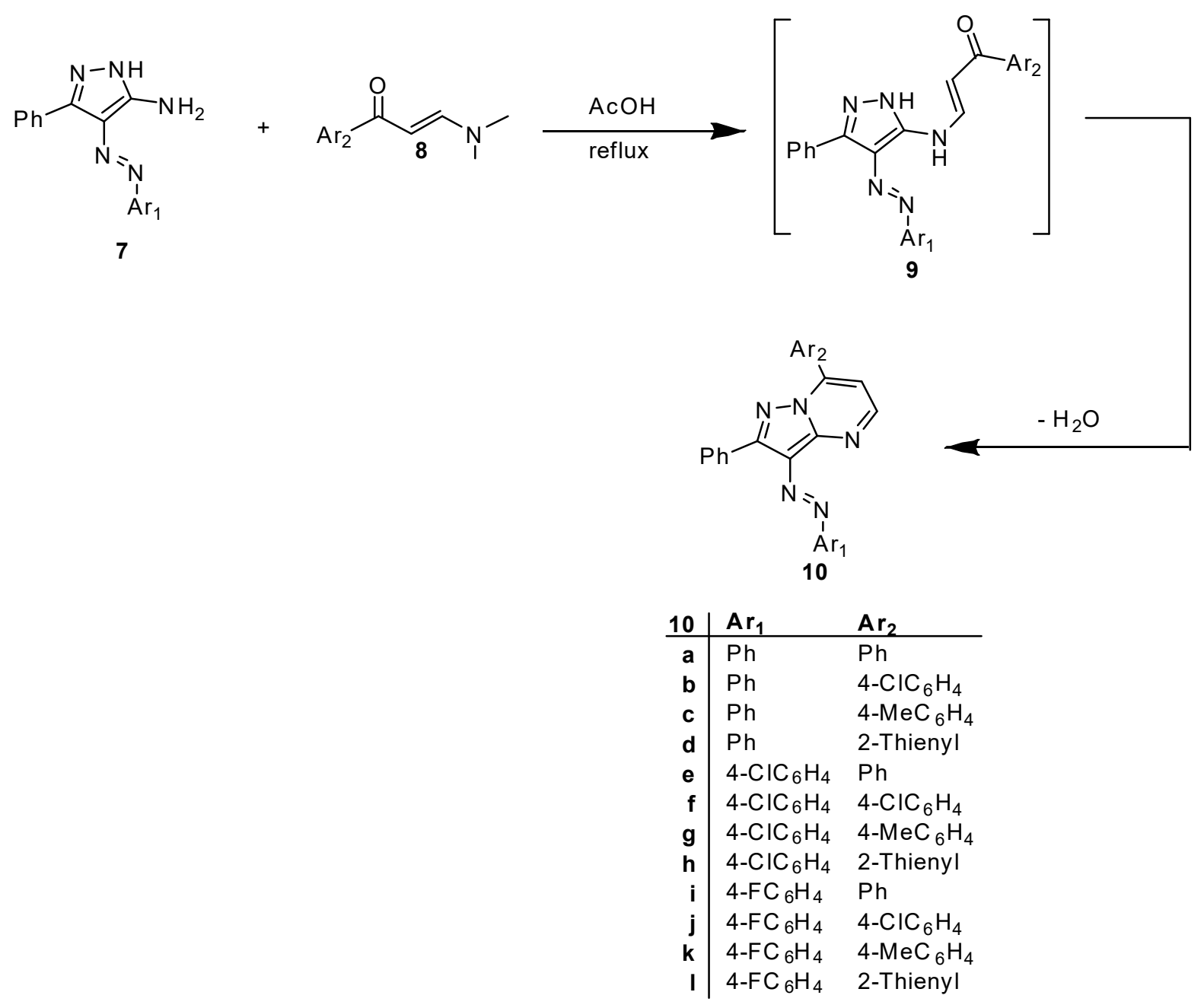

Scheme-2: Synthesis of Phenyldiazenyl pyrazolo[1, 5-a]pyrimidine Derivatives (10a-1)

Also, 5-amino phenyldiazenyl-1H-pyrazole (7a-c) were refluxed with 2-bromo acetophenone (2) in the presence of $\mathrm{K}_{2} \mathrm{CO}_{3}$ in acetone to afford the correspond imidazo[1,2-b]pyrazole derivatives (12a-c). The IR spectrum of compound 12a-c showed a sharp absorption band of NH at $3370 \mathrm{~cm}-1$. Also, the ${ }^{1} \mathrm{H}-\mathrm{NMR}$ spectrum of 12a-c exhibited $\mathrm{H}$ protons of $\mathrm{H}-3$ and $\mathrm{NH}$ of imidazo[1,2-b]pyrazole appear as singlet signals at $\delta \approx 8.45-8.50$ and $\delta \approx 12.32-12.78 \mathrm{ppm}$, respectively (Scheme-3).

\section{Biological Activity}

\section{Cytotoxicity Activity}

The attained results showed that all compounds had dose-dependent cytotoxicity against both cell types. (Table-1, Figs.-2 and 3). The constructed deduction from these outcomes is that, in assessment with the positive control doxorubicin, compounds $7 \mathbf{a}-\mathbf{c}$ with $\left(\mathrm{IC}_{50} \approx 29.4 \mu \mathrm{M}\right)$ and 10c-d $\left(\mathrm{IC}_{50} \approx 29.9 \mu \mathrm{M}\right)$ had comparable activities against HepG-2; compounds 10a, 10b, 10e, 10f, 10g, 10h, 10k, 10l, 12a and 12b had slightly moderate activities; compounds $\mathbf{1 2 c}, \mathbf{1 0} \mathbf{j}$ and $\mathbf{1 0} \mathbf{i}$, respectively, had fewer activities relative to the positive control, regarding human liver cancer (HepG-2) (Fig.-2 and Table-1). Regarding breast cancer cells (MCF-7); all the compounds were more potent relative to the positive control. In addition to 7a and 12a 
RASĀYAN J. Chem.

Vol. 14 | No. 2 |741-750| April - June | 2021

showed better anticancer activities against MCF-7 where $\mathrm{IC}_{50}$ for both compounds $\approx 6.1 \mu \mathrm{M}$ when compared with doxorubicin $\left(\mathrm{IC}_{50}=10.3 \mu \mathrm{M}\right)$ (Fig.-3 and Table-1).<smiles>CC(C)(C)c1ccccc1</smiles><smiles>[14CH3]N=Nc1c(-c2ccccc2)nn(CC(=O)c2ccccc2)c1N</smiles>

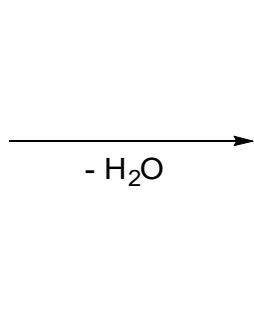<smiles>O=[W]N=Nc1c(-c2ccccc2)nn2cc(-c3ccccc3)[nH]c12</smiles>

Scheme-3: Synthesis of imidazo[1,2-b]pyrazole Derivatives (12a-c)

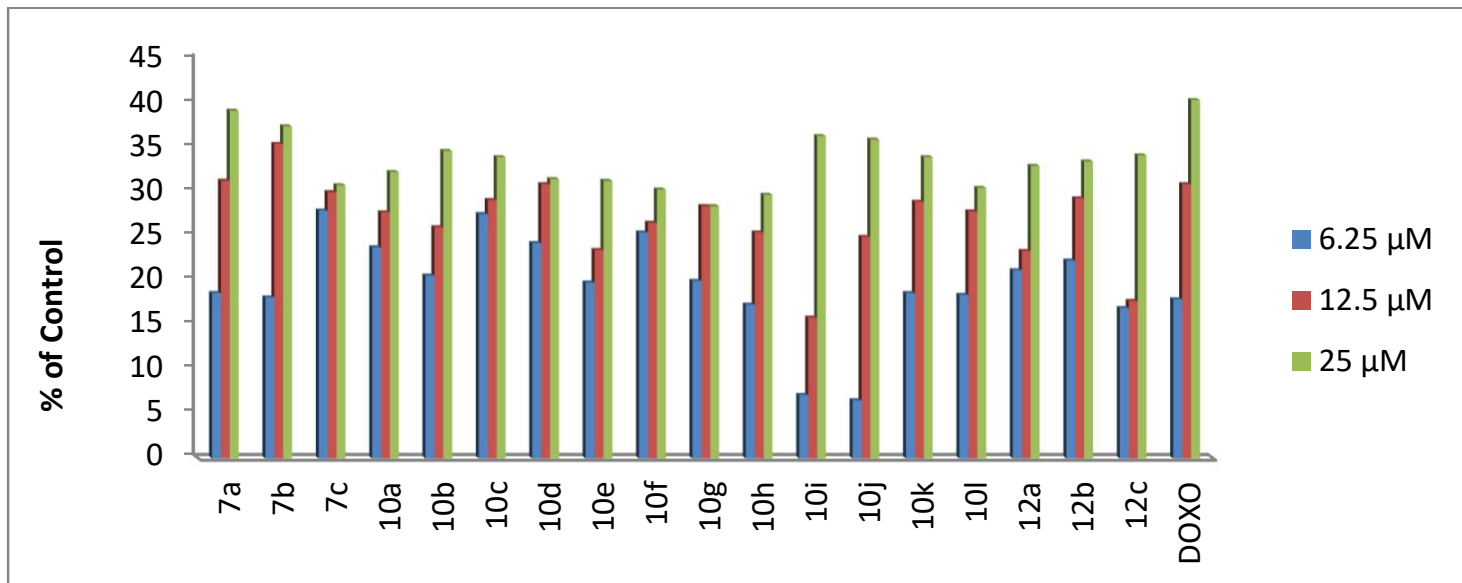

Fig.-2: The Compounds had Anticancer Activity against the HepG-2 Human Cancer Cell Line at Various Concentrations

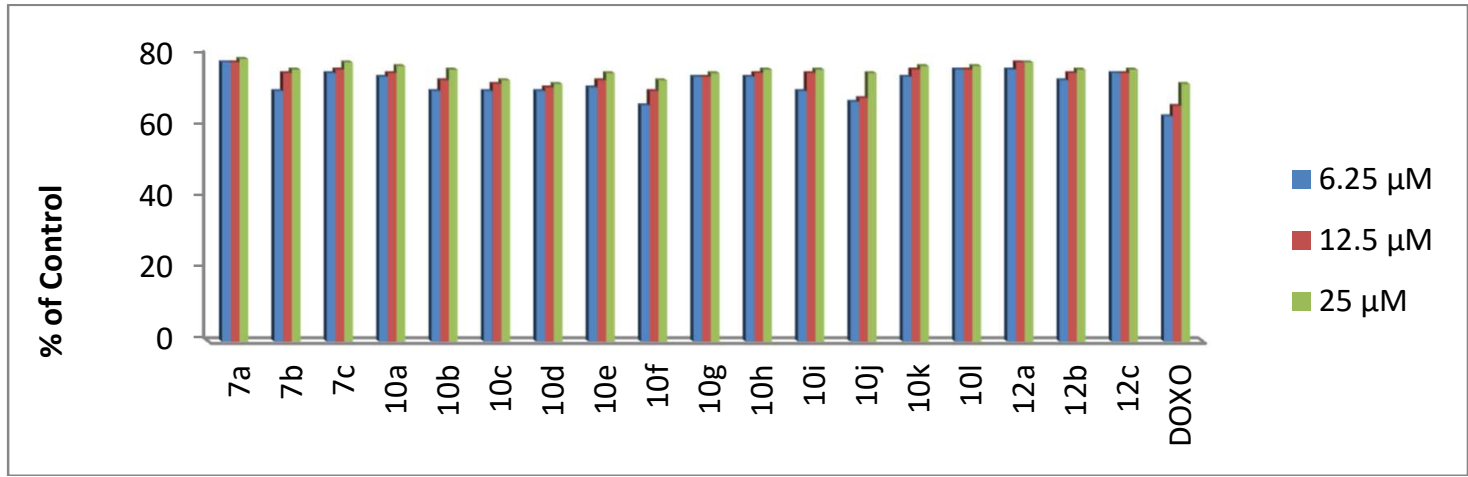

Fig.-3: The Compounds had Anticancer Activity against MCF-7 Human Cancer Cell Line at Various Concentrations

In general, from the SAR point of view, the pyrazolo [1-5a] pyrimidine and imidazo[1,2-b]pyrazole derivatives bearing phenyldiazenyl moiety have a significant anticancer agent. The combination of enaminones and 2-bromoacetophenone with 5-aminopyrazole are given benefits. The activity of methyl group and 2-thinyl afford 10c-d which exhibited the highest anticancer activity against A549 cancer cell, respectively. 
RASĀYAN J. Chem.

Vol. 14 | No. 2 |741-750| April - June | 2021

Table-1: The Cytotoxic $\mathrm{IC}_{50}$ Values of the Compounds were evaluated using the MTT Assay on Two Different Cell Lines

\begin{tabular}{c|c|c}
\hline \multirow{2}{*}{ Compound } & \multicolumn{2}{|c}{$\mathrm{IC}_{50}(\mu \mathrm{M}) \pm \mathrm{SD}$} \\
\cline { 2 - 3 } & HepG-2 & MCF-7 \\
\hline $7 \mathrm{a}$ & $29.6 \pm 2.9$ & $6.1 \pm 0.5$ \\
\hline $7 \mathrm{~b}$ & $29.2 \pm 3.1$ & $8.9 \pm 0.7$ \\
\hline $7 \mathrm{c}$ & $29.4 \pm 2.5$ & $8.5 \pm 0.4$ \\
\hline $10 \mathrm{a}$ & $30.5 \pm 1.9$ & $7.7 \pm 0.5$ \\
\hline $10 \mathrm{~b}$ & $31.1 \pm 2.6$ & $8.7 \pm 1.1$ \\
\hline $10 \mathrm{c}$ & $29.1 \pm 3.1$ & $9.5 \pm 1.1$ \\
\hline $10 \mathrm{~d}$ & $29.9 \pm 2.4$ & $9.1 \pm 0.9$ \\
\hline $10 \mathrm{e}$ & $32.5 \pm 3.1$ & $8.2 \pm 0.5$ \\
\hline $10 \mathrm{f}$ & $30.8 \pm 3.1$ & $9.6 \pm 0.8$ \\
\hline $10 \mathrm{~g}$ & $32.1 \pm 2.9$ & $8.7 \pm 1.1$ \\
\hline $10 \mathrm{~h}$ & $33.1 \pm 3.2$ & $9.6 \pm 0.6$ \\
\hline $10 \mathrm{i}$ & $36.7 \pm 3.5$ & $10.3 \pm 1.1$ \\
\hline $10 \mathrm{j}$ & $34.5 \pm 3.1$ & $7.5 \pm 0.4$ \\
\hline $10 \mathrm{k}$ & $31.0 \pm 2.8$ & $7.3 \pm 0.5$ \\
\hline 101 & $32.0 \pm 3.4$ & $6.1 \pm 0.4$ \\
\hline $12 \mathrm{a}$ & $31.9 \pm 2.7$ & $8.0 \pm 0.5$ \\
\hline $12 \mathrm{~b}$ & $30.3 \pm 2.5$ & $7.4 \pm 0.3$ \\
\hline $12 \mathrm{c}$ & $34.0 \pm 3.3$ & $10.3 \pm 0.8$ \\
\hline Doxorubicin & $28.5 \pm 1.9$ & \\
\hline & CONCLUSION & \\
\hline & &
\end{tabular}

New pyrimidine and imidazo[1,2-b] pyrazole derivatives bearing phenyldiazenyl moiety were built and screened for anticancer activity. A few compounds exhibited good anticancer activities against HepG-2 and MCF-7. The compounds 7a-c and 10c-d are the most potent compound against MCF-7 cancer cells.

\section{REFERENCES}

1. R.M. Aly, R. A. Serya, A. M. El-Motwally, A. Esmat, S. Abbas and D.A. Abou El Ella, Bioorganic Chemistry 75, 368(2017), DOI:10.1016/j.bioorg.2017.10.018

2. S.A. Ahmed, A.M. Hussein and W.G. Hozayen, Journal of Heterocyclic Chemistry, 44, 803(2007), DOI: $10.1002 /$ jhet. 5570440408

3. A. Gpalsamy, H. Yang, J.W. Ellingboe, H.R. Tsou, N. Zhang, E. Honores, D. Powell, M. Miranda, J.P. McGinnisb and S.K. Rabindran, Bioorganic and Medicinal Chemistry Letters, 15, 1591(2005), DOI: 10.1016/j.bmcl.2005.01.066

4. J. Zhang, J.F. Peng, T. Wang, P. Wang and Z.T. Zhang, Journal of Molecular Structure, 1120, 228(2016), DOI: 10.1016/j.molstruc.2016.05.026

5. A.M. Fahim and A.M. Farag. Journal of Molecular Structure 1199, 127025 (2020),DOI:10.1016/j.molstruc.2019.127025

6. M. S. Rao, T. B. Rao and C. P. Koteswara, Rasayan Journal of Chemistry 13(3), 1513(2020), DOI: 10.31788/RJC.2020.1335799

7. P. S. Parmar and S. K. Patel, Rasayan Journal of Chemistry 13(3), 1555(2020), DOI: 10.31788/RJC.2020.1335692

8. D. M. Sirsat, P. S. Bhale, H. V. Chavan, S. M. Karape and M. T. Bachute, Rasayan Journal of Chemistry 13, 1589(2020), DOI:10.31788/RJC.2020.1335768

9. V. P. Gilava1, P. K. Patel, H. K. Ram and J. H. Chauhan, Rasayan Journal of Chemistry, 13(4), 2249(2020), DOI: $10.31788 /$ RJC.2020.1346072

10. N. Petek, B. Štefane, M. Novinec and J. Svete, Bioorganic Chemistry 84, 226(2019), DOI: 10.1016/j.bioorg.2018.11.029

11. G.M. Ali, D.A. Ibrahima, A.M. Elmetwalia and Ismail. Bioorganic Chemistry, 86, 1(2019), DOI: $10.1016 /$ j.bioorg.2019.01.008 
RASĀYAN J. Chem.

Vol. 14 | No. 2 |741-750| April - June | 2021

12. P. Modi, S. Patel and M. Chhabria, Bioorganic Chemistry, 87, 240(2019), DOI:10.1016/j.bioorg.2019.02.044

13. A. Stefano, A. Anna, B. Maurizio, T. Alessandra, O. Francesco, S. Silvia, B. Chiara and Y. Matilde, ChemMedChem, 5, 1242(2010), DOI:10.1002/cmdc.201000165

14. A.E. Rashad, M.I. Hegab, R.E. Abdel-Megeid, J.A. Micky, and F.M. Abdel-Megeid, Bioorganic and Medicinal Chemistry Letters, 16, 7102(2008), DOI:10.1016/j.bmc.2008.06.054

15. D. Manetti, C. Brullo, M. Magnani, F. Mosci, B. Chelli, E. Crespan, S. Schenone, A. Naldini, O. Bruno and M.L. Trincavelli, Journal of Medcinal Chemistry, 51, 1252(2008), DOI:10.1021/jm701240c

16. M. Radi, E. Dreassi, C. Brullo, E. Crespan, C. Tintori, V. Brenardo, M. Valoti, C. Zamperini, H. Daigi, F. Musumeci and F. Carraro, Journal of Medicinal Chemistry, 54, 2610(2011), DOI: $10.1021 / \mathrm{jm} 1012819$

17. A.S. Hassan, A.F. Mady, H.M. Awad and T.S. Hafez, Chinese Chemical Letters, 28, 388(2017), DOI: $10.1016 /$ j.cclet.2016.10.022

18. A.S. Hassan, T.S. Hafez, S.A. Osman and M.M. Ali, Turkish, Journal of Chemistry, 39, 1102(2015), DOI:10.3906/kim-1504-12

19. N.S. Ismail, G.M. Ali, D.A. Ibrahim and A.M. Elmetwali, Future Journal of Pharmaceutical Science, 2, 60(2016), DOI:10.1016/j.fjps.2016.08.004

20. N.R. Kumar, Y. Poornachandra, D.K. Swaroop, G.J. Dev, C.G. Kumar and B. Narsaiah, Bioorganic and Medicinal Chemistry Letters, 26, 5203(2016), DOI:10.1016/j.bmcl.2016.09.062

21. O.M. Ahmed, M.A. Mohamed, R.R. Ahmed and S.A. Ahmed, European Journal of Medicinal Chemistry, 44, 3519(2009), DOI:10.1016/j.ejmech.2009.03.042

22. E.K. Abdelall and J.N. Philoppes, ARKIVOK v, 210 (2016), DOI:10.3998/ark.5550190.p009.743

23. T.A. Farghaly and A.S. Shawali Tetrahedron, 66, 2700 (2010), DOI:10.1016/j.tet.2010.02.012

24. A. Rahmati, M.E. Vashareh and M.A. Kouzehrash, Tetrahedron, 69, 4199(2013), DOI:10.1016/j.tet.2013.03.103

25. J. Khalafy, A.P. Marjani F. Salami. Tetrahedron Letters, 55, 6671(2014), DOI: 10.1016/j.tetlet.2014.10.061

26. A.T. Baviskar, C. Madaan, R. Preet, P. Mohapatra, V. Jain, A. Agarwal, S.K. Guchhait, C.N. Kundu, U.C. Banerjee and P.V. Bharatam, Journal of Medicinal Chemistry, 54, 5013(2011), DOI:10.1021/jm200235u

27. S. Grosse, V. Mathieu, C. Pillard, S. Massip, M. Marchivie, C. Jarry, P. Bernard, R. Kiss and G. Guillaumet, European Journal of Medicinal Chemistry, 84,718(2014), DOI: 10.1016/j.ejmech.2014.07.057

28. A.F. Kassem, I.F. Nassar, M.T. Abdel-Aal, H.M. Awad and W.A. El-Sayed, Chemical and Pharmaceutical Bulletin, 67, 888(2019), DOI:10.1248/cpb.c19-00280

29. F.M. Alminderej, H.H. Elganzory, M.N. El-Bayaa, H.M Awad and W.A. El-Sayed, Molecules, 24, 3738(2019), DOI: 10.3390/molecules24203738

30. M.E. Haiba, E.S. Al-Abdullah, N.S. Ahmed, H.A. Ghabbour, H.M .Awad, Journal of Molecular Structure, 1195, 702(2019), DOI:10.1016/j.molstruc.2019.05.081

[RJC-6137/2020] 